\title{
p53 Promotes Differentiation of Cardiomyocytes from hiPSC through Wnt Signaling-Mediated Mesendodermal Differentiation
}

\author{
Yuanshu Liu ${ }^{1, *}$, Peng Zhang ${ }^{3, *}$, Wenjun Huang ${ }^{1,2}$, Feng Liu ${ }^{1}$, Dandan Long ${ }^{1}$, Wanling Peng ${ }^{1}$, \\ Xitong Dang ${ }^{1}$, Xiaorong Zeng ${ }^{1}$, Rui Zhou ${ }^{1,2}$
}

\begin{abstract}
${ }^{1}$ The Key Laboratory of Medical Electrophysiology of Ministry of Education and Medical Electrophysiological Key Laboratory of Sichuan Province, Collaborative Innovation Center for Prevention and Treatment of Cardiovascular Disease of Sichuan Province, Institute of Cardiovascular Research, Southwest Medical University, Luzhou, China

${ }^{2}$ Shaanxi Institute for Pediatric Diseases, Xi'an Key Laboratory of Children's Health and Diseases, Department of Cardiology, Xi'an Children's Hospital, Affiliated Children's Hospital of Xi'an Fiaotong University, Xi'an, China

${ }^{3}$ Department of Anesthesiology, Sichuan Academy of Medical Science E Sichuan Provincial People’s Hospital, University of Electronic Science and Technology of China, Chengdu, China
\end{abstract}

Background and Objectives: Manipulating different signaling pathways via small molecules could efficiently induce cardiomyocytes from human induced pluripotent stem cells (hiPSC). However, the effect of transcription factors on the hiPSC-directed cardiomyocytes differentiation remains unclear. Transcription factor, p53 has been demonstrated indispensable for the early embryonic development and mesendodermal differentiation of embryonic stem cells (ESC). We tested the hypothesis that p53 promotes cardiomyocytes differentiation from human hiPSC.

Methods and Results: Using the well-characterized GiWi protocol that cardiomyocytes are generated from hiPSC via temporal modulation of Wnt signaling pathway by small molecules, we demonstrated that forced expression of p53 in hiPSC remarkably improved the differentiation efficiency of cardiomyocytes from hiPSC, whereas knockdown endogenous p53 decreased the yield of cardiomyocytes. This p53-mediated increased cardiomyocyte differentiation was mediated through WNT3, as evidenced by that overexpression of 553 upregulated the expression of WNT3, and knockdown of p53 decreased the WNT3 expression. Mechanistic analysis showed that the increased cardiomyocyte differentiation partially depended on the amplified mesendodermal specification resulted from p53-mediated activation of WNT3-mediated Wnt signaling. Consistently, endogenous WNT3 knockdown significantly ameliorated mesendodermal specification and subsequent cardiomyocyte differentiation.

Conclusions: These results provide a novel insight into the potential effect of p53 on the development and differentiation of cardiomyocyte during embryogenesis.

Keywords: hiPSC, Cardiomyocytes, p53, Wnt signaling

Received: March 13, 2021, Revised: May 11, 2021, Accepted: May 17, 2021, Published online: June 30, 2021

Correspondence to Rui Zhou

Shaanxi Institute for Pediatric Diseases, Affiliated Children's Hospital of Xi'an Jiaotong University, No. 69, Xijuyuan Lane, Lianhu District, Xi'an 710003, China

Tel: +86-029-87692102, Fax: +86-029-87692102, E-mail: zhouhuaxizhu@126.com

Co-Correspondence to Xiaorong Zeng

Institute of Cardiovascular Research, Southwest Medical University, 3-319 Zhongshan Road, Luzhou 646000, China

Tel: +86-0830-3160619, Fax: +86-0830-3160619, E-mail: zxr8818@vip.sina.com

${ }^{*}$ These authors contributed equally to this work.

다 This is an open-access article distributed under the terms of the Creative Commons Attribution Non-Commercial License (http://creativecommons.org/licenses/by-nc/4.0/), which permits unrestricted non-commercial use, distribution, and reproduction in any medium, provided the original work is properly cited.

Copyright (c) 2021 by the Korean Society for Stem Cell Research 


\section{Introduction}

Heart disease is a leading cause of morbidity and mortality. Loss of cardiomyocytes is one of the main pathological features in most heart diseases, which initiates fibrosis and scar formation and eventually leading to heart failure. However, the very limited ability of human adult cardiomyocytes (CMs) to regenerate significantly hampered the development of novel therapeutic strategies. Human-induced pluripotent stem cell (hiPSC)derived cardiomyocytes could potentially provide an unlimited supply of cardiomyocytes (1).

A series of different monolayer-based protocols for cardiomyocytes differentiation from hiPSC have been established, where small molecules and growth factors were used to modulate signaling pathways, such as Activin/ Nodal, BMP, Wnt and TGF $\beta$, to induce cardiac lineage differentiation following the stages of hiPSC, mesendoderm, mesoderm, cardiac mesoderm, cardiac progenitors and finally to induced cardiomyocytes, a process similar to the in-vivo embryonic heart development (2-5). For example, sequential exposure to activin A and BMP4 could generate more than $30 \%$ cardiomyocytes from the hiPSC $(2,6)$. Lian et al. (7) further reported that sequential activation and inactivation of Wnt signaling in the absence of exogenous activin A and BMP4 was sufficient to efficiently produce around 90\% cardiomyocytes from hiPSC. However, the roles of transcription factors on the hiPSC-directed cardiomyocytes differentiation remains unexplored.

The transcription factor p53, a well-known tumor suppressor, plays a crucial role in early embryo development and differentiation (8-12). Unlike quite low level kept by complex regulatory mechanisms in terminal differentiated somatic cells, p53 is expressed at relatively high levels in mouse embryo germ layer progenitors and embryonic stem cells (ESCs), suggesting its potential functions in ESCs and early development $(8,9)$. Xenopus embryos failed to undergo gastrulation after p53 depletion (10). Monolayer culture of mouse embryonic stem cells (ESC) requires p53 for mesendodermal differentiation $(11,12)$. Activation of the p53 pathway suppressed iPSC generation $(13,14)$, whereas p53 reactivation in teratocarcinomas promotes cancer cell differentiation (15), suggesting the promoting effect of p53 on differentiation. It has been demonstrated that $\mathrm{p} 53$ activates multiple TGF- $\beta$ target genes via binding to Smad proteins during xenopus embryonic development (16). Using the chromatin immunoprecipitationbased microarray (ChIP-chip) and gene expression microarray assays, Lee et al. (17) revealed that the expression of Wnt ligand genes was strongly induced in a p53-dependent manner in mESC upon treatment with DNAdamaging agents. All these observations point to the possibility that p53 may function in the hiPS-directed cardiomyocytes differentiation.

In this study, we investigated whether the transcription factor p53 could promote the differentiation of cardiomyocytes from hiPSC. We found that p53 remarkably promoted the efficiency of cardiomyocyte differentiation from hiPSC based on modulation of Wnt signaling pathway by small molecules, which was partially attributed to the amplified mesendodermal specification resulted from increased expression of Wnt signaling gene, WNT3. These results provide a novel mechanistic insight into the effect of p53 on the development and differentiation of embryonic heart.

\section{Materials and Methods}

\section{Materials}

The information of commercial reagents and cell lines used in the study are listed below: LHpb-YaabC3 hiPSC (HNF-P30-P11, OSINGLAY BIO, China), HN4 human embryonic stem cell (hESC) line (HES-P20-P9, OSINGLAY BIO, China), RPMI/1640 media (11875093, Thermo Fisher Scientific, USA), DMEM/F12 media (11320082, Thermo Fisher Scientific, USA), BioCISO medium for hiPSC (BC-PM0001, OSINGLAY BIO, China), Glycogen synthase kinase 3 (GSK3) inhibitor CHIR99021 (S1263, Sigma, USA), Wnt inhibitor IWP2 (3533, Tocris Bioscience, UK), ROCK inhibitor Y27632 (1254, Tocris Bioscience, UK), B-27 supplement with (17504044, Thermo Fisher Scientific, USA) or without insulin (A1895601, Thermo Fisher Scientific, USA), Matrigel for ESC (354277, Corning, UK), TRIzol Reagent (15596026, Thermo Fisher Scientific, USA), Real-time PCR reagents (208056, Qiagen, Germany). Propidium Iodide (PI) Staining Solution (556463, BD Biosciences, USA). All lentiviruses overexpressing human P53 and knocking down P53 and WNT3 using shRNA technology were designed and packaged by GENE CHEM, China. All primers/oligos were synthesized by Sangon Biotech, China and listed in Supplementary Table S1. All other reagents, unless specified otherwise, were products of Sigma.

\section{Cell culture and lentivirus transduction}

Both HN4 and hiPSC were maintained in the hPSC BioCISO medium in plates pre-coated with Matrigel (1 : 50). When reaching $80 \sim 90 \%$ confluence, cells were trypsinized and passaged in a $1: 4 \sim 7$ ratio using BioCISO me- 
dium supplemented with $5 \mu \mathrm{M}$ Y27632. After $24 \mathrm{~h}$, cells were transduced with lentiviruses at MOI $1: 50$ in the presence of $8 \mu \mathrm{g} / \mathrm{ml}$ of polybrene and $1 \mu \mathrm{g} / \mathrm{ml}$ of puromycin for stable cloning. The overexpressing P53 and knocking down P53 and WNT3 were identified by qRT-PCR and Western blot.

\section{Cardiomyocytes differentiation from hiPSC}

The cardiac induction from hiPSC was performed in a growth factor- and serum-free system by temporal modulation of canonical Wnt signaling, GSK3 inhibitor (Gi) and Wnt inhibitor (Wi), where highly efficient differentiation of cardiomyocytes was enabled through sequential temporal Wnt activation by Gi and Wnt inhibition by Wi, the GiWi protocol (3). At about 80 90\% confluent, hiPSC cultures were split into a 12 -well plate pre-coated with Matrigel at $1 \times 10^{5}$ cells per well containing $2 \mathrm{ml}$ of hPSC medium on day -4 . At day 0 the medium was changed to RPMI/B-27 minus insulin with CHIR99021 (10 $\mu \mathrm{M}$, GSK3 inhibitor). At day 1 the medium was refreshed by RPMI/B-27 without insulin for $48 \mathrm{~h}$. At day 3 of differentiation (72 h after addition of CHIR99021), RPMI/B-27 minus insulin with IWP2 (5 $\mu \mathrm{M}$, Wnt inhibitor) was added for $48 \mathrm{~h}$ followed by RPMI/B-27 minus insulin refreshing from day 5 to 7 . On day 7 of differentiation and every 3 days thereafter, RPMI/B-27 with insulin was refreshed. The beating cardiomyocytes were observed as early as on day 8 .

\section{RNA isolation and qPCR}

Total RNA was isolated using TRIzol method. One $\mu \mathrm{g}$ of total RNA was reversely transcribed in a total volume of $10 \mu \mathrm{l}$ with ReverTra Ace qPCR RT Master Mix kit (FSQ-201, TOYOBO, Japan) following manufacturer's instructions. One $\mu 1$ of three-times diluted cDNA was used for real-time PCR in a $20 \mu 1$ reaction using SYBR Green Real Time PCR Mix (204143, Qiagen, Germany). The PCR conditions were $95^{\circ} \mathrm{C}$ for $2 \mathrm{~min}$, followed by 40 cycles of $95^{\circ} \mathrm{C}$ for $20^{\prime \prime}$ and $60^{\circ} \mathrm{C}$ for $15^{\prime}$. All primers used were listed in Supplementary Table S1. The expression of target gene was normalized to GAPDH and calculated using $2^{-\Delta \Delta \mathrm{Ct}}$ method as described previously (18).

\section{Immunofluorescence assay}

Well-suspended hiPSC cells and induced cardiomyocytes were seeded in a $\mu$-Slide 8 well (80827, ibidi, USA) pre-coated with Matrigel $(1: 100)$ at the density of $2 \times 10^{4}$ per well. $48 \mathrm{~h}$ later cells were fixed with $4 \%(\mathrm{w} / \mathrm{v})$ Paraformaldehyde (PFA) for $15 \mathrm{~min}$ at room temperature, permeabilized, and incubated with following first primary antibodies: anti-OCT4 antibody (\#2750, Cell Signaling Technology, USA), anti-NANOG antibody (\#3580, Cell Signaling Technology, USA), anti-SSEA4 antibody (\#4755, Cell Signaling Technology, USA), anti-TRA-1-60 antibody (\#4746, Cell Signaling Technology, USA), anti-MKI67 antibody (ab15580, abcam, USA), anti-NKX2-5 antibody (ab91196, abcam, USA), anti-cTNT antibody (MS-295-P1, Thermo Fisher Scientific, USA) and anti- $\alpha$ ACTININ antibody (A7811, Sigma, USA), followed by their corresponding fluorescence-labeled secondary antibodies, alexa fluor 488 labeled goat anti-rabbit IgG (A-11008, Invitrogen, USA), alexa fluor 488 labeled goat anti-mouse IgG (A-11001, Invitrogen, USA), alexa fluor 594 labeled goat anti-rabbit IgG (R37177, Invitrogen, USA) and alexa fluor 594 labeled goat anti-mouse IgG (A-11005, Invitrogen, USA). The slides were then counterstained using $0.5 \mu \mathrm{g} / \mathrm{ml}$ of DAPI (\#4083, Cell Signaling Technology, USA) for $15 \mathrm{~min}$ at room temperature. After rinsing with PBS, the chambers were mounted and visualized by fluorescence microscopy (IX83, Olympus, Japan).

\section{Flow cytometry}

For cardiomyocytes identification, the induced cardiomyocytes from hiPSC in 12-well plate were dissociated into single cells using $0.25 \%$ trypsin with $0.5 \mathrm{mM}$ EDTA followed by washing with PBS. Cells were fixed in formaldehyde at a final concentration of $4 \%$ for $10 \mathrm{~min}$ at RT and chilled on ice for $1 \mathrm{~min}$. Permeabilization was performed by adding ice-cold $100 \%$ methanol slowly to pre-chilled cells to a final concentration of $90 \%$ methanol followed by incubation on ice for $30 \mathrm{~min}$. Permeabilized cells were then blocked with blocking buffer $(0.5 \%$ BSA in PBS) for $10 \mathrm{~min}$, incubated with anti-cTNT antibody (MS-295-P1, Thermo Fisher Scientific, USA) for $1 \mathrm{~h}$, and followed by incubation with alexa fluor 488 labeled goat anti-mouse IgG (A-11001, Invitrogen, USA) for $30 \mathrm{~min}$ at room temperature. Samples were analyzed using Flow cytometry machine (BD FACSVerse, BD Bioscience, USA) according to the manufacturer's protocol. All the data was analyzed using FlowJo V10.0 software.

For cell cycle analysis, 80 90\% confluent hiPSC in 6-well plate were dissociated into single cells using $0.25 \%$ trypsin with $0.5 \mathrm{mM}$ EDTA. After being washed with ice-cold PBS, cells were fixed in cold $70 \%$ ethanol for 30 minutes, and washed twice with PBS. Cells were spun down, resuspended with staining buffer consisting of 100 $\mu \mathrm{g} / \mathrm{ml} \mathrm{RNase} \mathrm{A}$ and $25 \mu \mathrm{g} / \mathrm{ml}$ propidium iodide (PI), and continued to incubate for $30 \mathrm{~min}$ at RT. Samples were analyzed using flow cytometry machine (BD FACSVerse, 
BD Bioscience, USA) according to the manufacturer's protocol. All the data was analyzed using FlowJo V10.0 software.

\section{Western blot}

Western blot was performed following the protocol reported previously (18). Briefly, cells were lysed using RIPA lysis buffer supplemented with proteinase inhibitor cocktail (78430, Thermo Fisher Scientific, USA), sonicated and boiled for $10 \mathrm{~min}$. Lysates were spun down at top speed for $1 \mathrm{~min}$ at room temperature and supernatants were quantitated using a BCA Protein Assay Kit (71285, Merk, USA). NE-PER ${ }^{\mathrm{TM}}$ Nuclear and Cytoplasmic Extraction Reagents (78833, Thermo Fisher Scientific, USA) was used for nuclear protein extraction according to vendor's instructions. Twenty-five $\mu \mathrm{g}$ of protein was resolved with $12.5 \%$ SDS-PAGE, and transferred onto PVDF membrane (Millipore, USA). The membrane was blocked with $5 \%$ milk in TBST for 1 hour at RT, probed with following first antibodies for overnight at $4^{\circ} \mathrm{C}$ : anti-p53 antibody (\#9282, Cell Signaling Technology, USA), anti-OCT4 antibody (\#2750, Cell Signaling Technology, USA), anti-NANOG antibody (\#3580, Cell Signaling Technology, USA), anti-WNT3 antibody (ab28472, Abcam, USA), anti- $\beta$-Catenin Antibody (\#9562, Cell Signaling Technology, USA), anti-Histone H3 Antibody (\#9715, Cell Signaling Technology, USA), and anti-GAPDH antibody (\#2118, Cell Signaling Technology, USA), washed, and followed by incubation with their corresponding secondary antibodies for 1 hour at room temperature. The membrane was then developed with highly sensitive ECL luminescence reagent (C500044, Sangon Biotech, China), and images were acquired. Relative expression of total and nuclear protein, normalized to that of GAPDH and Histone $\mathrm{H} 3$ respectively, was analyzed by densitometry using the Molecular Analyst software (Imaging Densitometer, Bio-Rad).

\section{Statistical analysis}

GraphPad Prism 8.0 software (GraphPad, USA) was used to perform statistical analysis and data with at least three independent experiments are presented as the mean \pm SD. Comparisons between two groups were performed using one-way analysis of variance (one-way ANOVA), with $\mathrm{p}<0.05$ was considered statistically significant.

\section{Results}

\section{Characterization of hiPSC}

hiPSC colonies displayed typical morphology such as high nucleus-to-cytoplasm ratio and prominent nucleoli morphology (Fig. 1A). The hiPSC expressed high levels of pluripotency markers OCT4, SOX2, NANOG and KLF4 that were similar to that of hESC line, HN4 (Fig. 1B). Immunofluorescence (IF) showed high-intensity fluorescence signals localized in nuclei (OCT4 and NANOG) and on membrane (SSEA4 and TRA-1-60) respectively (Fig. 1C). In addition, the expression of cell proliferation markers, AURKB and MKI67 in hiPSC was similar to that in hESC (Fig. 1D). The high level of MKI67 expression was further confirmed by IF (Fig. 1E). These results indicate that the hiPSC possesses pluripotency and self-renewal capacity.

\section{Identification of hiPSC-induced cardiomyocytes}

Schematic protocol for cardiomyocyte differentiation from hiPSC was shown (Fig. 2A), where temporal modulation of canonical Wnt signaling, the GiWi protocol (sequential activation (Gi) and inactivation of Wnt (Wi) signaling pathways) directs the differentiation of hiPSC into beating cardiomyocytes (3). The differentiating cardiomyocytes showed time-dependent increase of cardiac progenitor maker NKX2-5 and cardiomyocyte marker TNNT2 (Fig. 2B). On day 21, IF showed nuclear localization of NXK2-5 and cytosolic localization of cTNT and $\alpha$-ACTININ (Fig. 2C). Noticeably the staining of cTNT and $\alpha$-ACTININ displayed characteristic striations indicating relatively mature sarcomere in some clusters of cardiomyocytes (Fig. 2C). Together with the movie showing a high percentage of beating cardiomyocytes with regular rhythm (Video was not shown), the results suggest successful cardiomyocytes differentiation from hiPSC via the GiWi protocol that was therefore used to generate cardiomyocytes for all experiments thereafter.

\section{Effect of p53 overexpression on the characteristics of hiPSC}

In order to test the effects of p53 on hiPSC, cells were transduced with lenti-P53 and lenti-control. Successful transduction of both lenti-P53 and lenti-control were indicated by high percent of $\mathrm{GFP}^{+}$hiPSC (Fig. 3A). Positive hiPSC cells overexpressing P53 were further validated by qRT-PCR (Fig. 3B) and Western blot (Fig. 3C and 3D). Characterization of hiPSC-P53 showed that there was no significant differences between lenti-P53 and lenti-control group in terms of the expression of the pluri- 


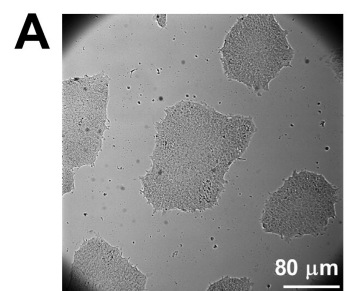

B
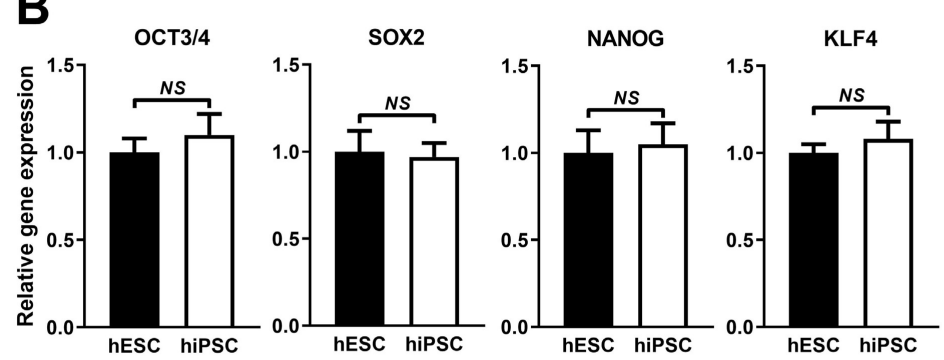

C
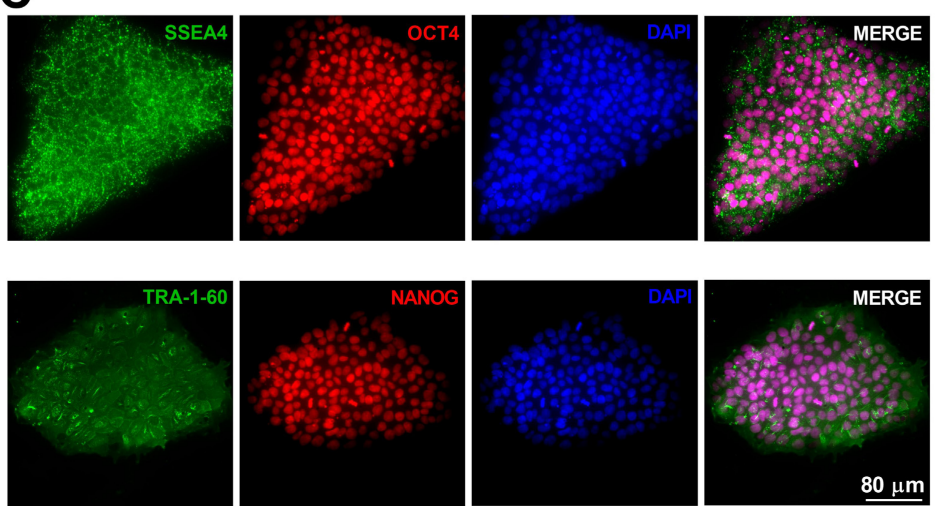

D

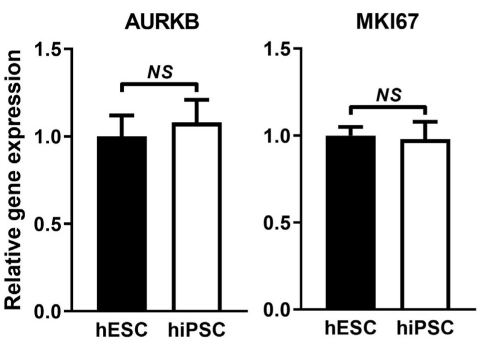

$E$
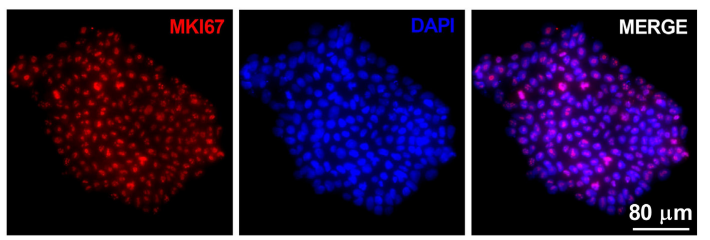

Fig. 1. Identification of the hiPSC. (A) Representative images displayed the typical morphology of hiPSC. (B) qRT-PCR results showed hiPSC has similar mRNA levels to the human embryonic stem cells (hESC) regarding the pluripotency marker genes (OCT4, SOX2, NANOG and KLF4). (C) Pluripotency markers of hiPSC (OCT4, NANOG, SSEA4 and TRA-1-60) were confirmed by immunofluorescence (IF) assay. (D) qRT-PCR results showed that hiPSC has similar mRNA levels to the hESC regarding the proliferation marker genes, MKI67 and AURKB. (E) MKI67 was further identified using IF method. Expression values of the PCR analysis were normalized to GAPDH. Data are presented as mean $\pm S D$ (t test, NS not significant; $n=3$ ).

potency marker genes (OCT4, SOX2, NANOG and KLF4) (Fig. 3E), and of the proliferation markers (AURKB and MKI67) (Fig. 3H), by qRT-PCR. However, forced expression of P53 slightly decreased the expression of NANOG with statistical significance, but not OCT4 at protein levels, compared to control (Fig. 3F and 3G). Flow cytometry analysis further showed that overexpression of P53 did not affect the cell cycle distribution of G0/G1, S and G2 phase (Fig. 3I and 3J). Taken together, our observations indicate that P53 overexpression does not significantly affect the characteristics of hiPSC regarding the pluripotency, self-renewal capacity and cell cycle distribution.

\section{p53 promotes the differentiation of hiPSC toward cardiomyocytes}

The effects of $\mathrm{p} 53$ on promoting the differentiation of hiPSC toward cardiomyocytes was then investigated. Forced expression of p53 remarkably increased the mRNA levels of cardiomyocyte markers TNNT2, TNNI3 and $\alpha$ -ACTNIN (Fig. 4A) and of cardiac progenitor marker NKX2-5 (Fig. 4B) on day 21 after differentiation. Flow cytometry showed that the percentage of $\mathrm{cTnT}^{+}$cardiomyocytes was significantly higher in hiPSC-P53 than the control on day 21 (Fig. 4C and 4D). It is well-established that the process of the GiWi-based differentiation of hiPSC toward cardiomyocytes mimics the natural embryonic heart development processes, starting from embryonic stem cells to mesendoderm, cardiac mesoderm, car- 
A

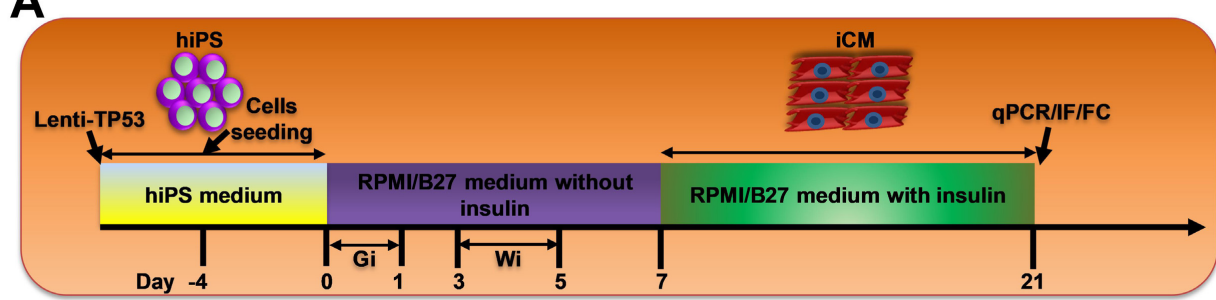

\section{B}

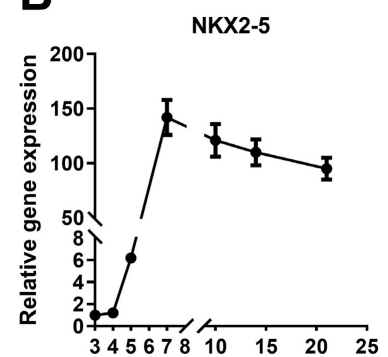

TNNT2

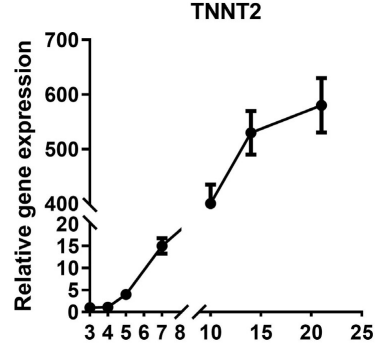

C
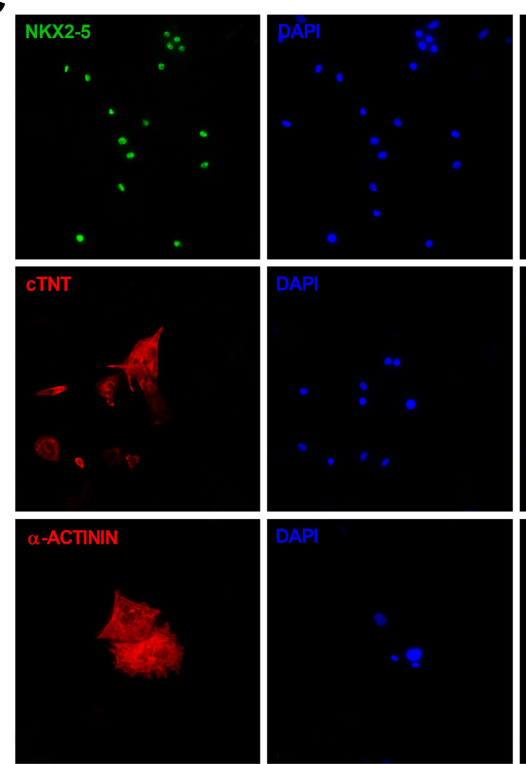

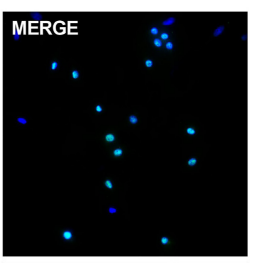

MERGE
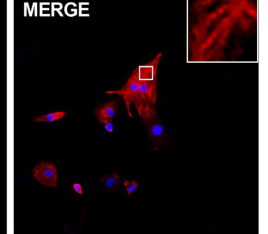

MERGE

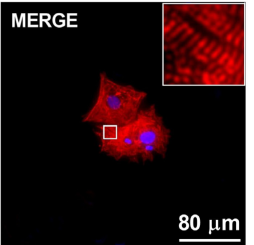

Fig. 2. Identification of the hiPSC derived cardiomyocytes. (A) Schematic illustration of an experimental protocol for the cardiomyocytes differentiation from hiPSC via sequential transient modulation of canonical Wnt signaling pathway using GiWi method. Gi, GSK3 inhibitor; Wi, Wnt inhibitor; $\mathrm{ICM}$, induced cardiomyocytes; IF, immunofluorescence; FC, flow cytometry. (B) Time course analysis of the expression of cardiac progenitor marker (NKX2-5) and cardiomyocytes marker (TNNT2) was performed using qRT-PCR assay. (C) The protein of cardiac progenitor marker (NKX2-5) and cardiomyocytes marker (CTNT and $\alpha$-ACTININ) was further evaluated by IF method. Data are presented as mean \pm SD. diac progenitor cells, and finally to cardiomyocytes. To test our hypothesis that forced expression of $\mathrm{p} 53$ boosts the differentiation of cardiomyocytes at earlier stages rather than cardiac progenitor stage, markers of mesendoderm and cardiac mesoderm were analyzed by qRT-PCR on day 3 and 5. As expected, both mesendoderm indicators BRACHYURY and EOMES (Fig. 4E), and mesoderm markers MESP1 and KDR (Fig. 4F) were consistently increased by P53 overexpression compared to the standard GiWi protocol. These results suggest that p53 enhances the cardiomyocytes-differentiation program at as early as mesendoderm stage.

\section{Knocking down P53 in hiPSC suppresses its differentiation toward cardiomyocytes}

We have shown that p53 overexpression increased cardiomyocytes yields from hiPSC. To further investigate the role of p53 on the differentiation of hiPSC toward cardiomyocyte, endogenous p53 was knocked down by lenti-sh-TP53. Successful knockdown of TP53 in hiPSC (Fig. $5 \mathrm{~A} \sim \mathrm{C})$ significantly reduced mRNA levels of cardiac marker TNNT2 (Fig. 5D) and progenitor marker NKX2-5 (Fig. 5E) in the induced cardiomyocytes in comparison to that in the control, which was further confirmed by flow cytometry showing a significantly decreased percentage of cTNT $^{+}$cardiomyocytes on day 21 in hiPSC-sh-TP53 compared to control (Fig. 5F and 5G). Moreover, knockdown of TP53 caused significant decrease of mRNA levels of both mesendoderm indicators, BRACHYURY and EOMES (Fig. 5H), and mesoderm markers, MESP1 and $\mathrm{KDR}$ (Fig. 5I) on day 3 and 5 from differentiation respectively. Taking the crucial role of p53 in the mesendodermal specification into account $(11,12)$, other lineage markers regarding the germ layers were investigated using qRT-PCR assay (Supplementary Fig. S1). Consistently, the mesendodermal marker genes (GSC, FOXA2, MIXL1 and SOX17) were remarkably decreased by Si-TP53 (Supplementary Fig. S1A) in addition to BRACHYURY and EOMES (Fig. 5H). In contrast, the ectodermal markers (PAX6, NES and SOX1) were significantly up-regulated by knockdown of TP53 gene (Supplementary Fig. S1B), suggesting that in addition to the promoted effect of mesendodermal differentiation, p53 exerts the blocking effect of ectodermal specification as well. 
A

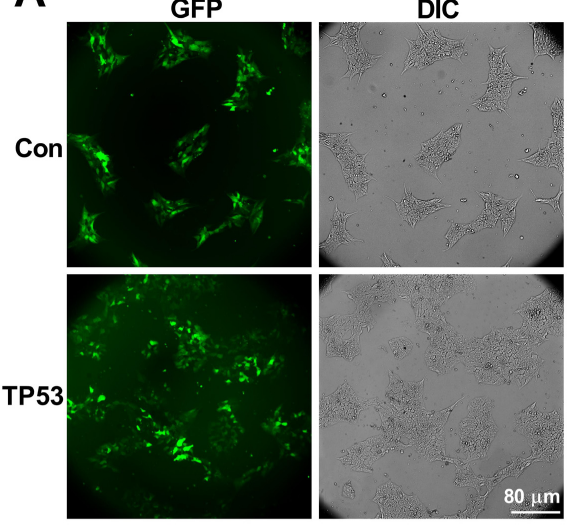

E
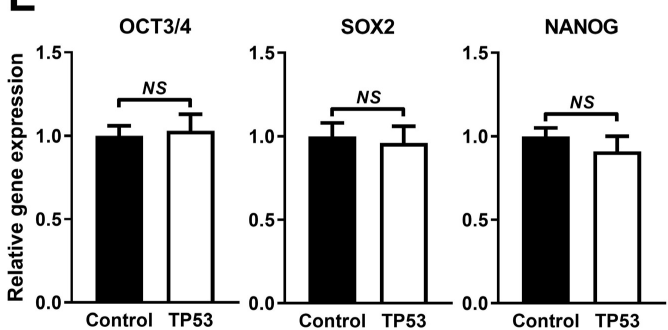

H
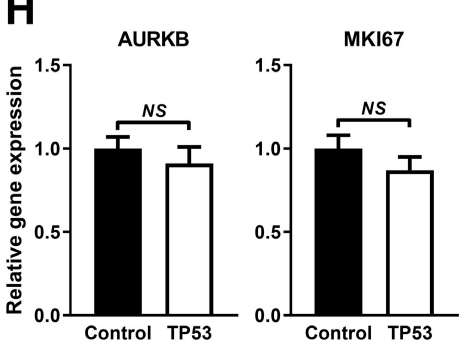

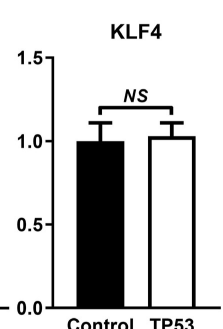

B

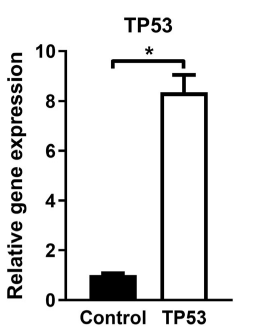

I

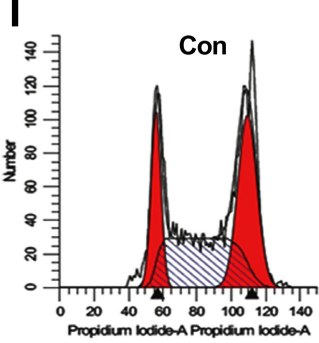

$\mathbf{F}$

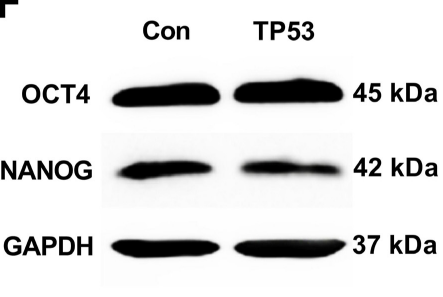

C
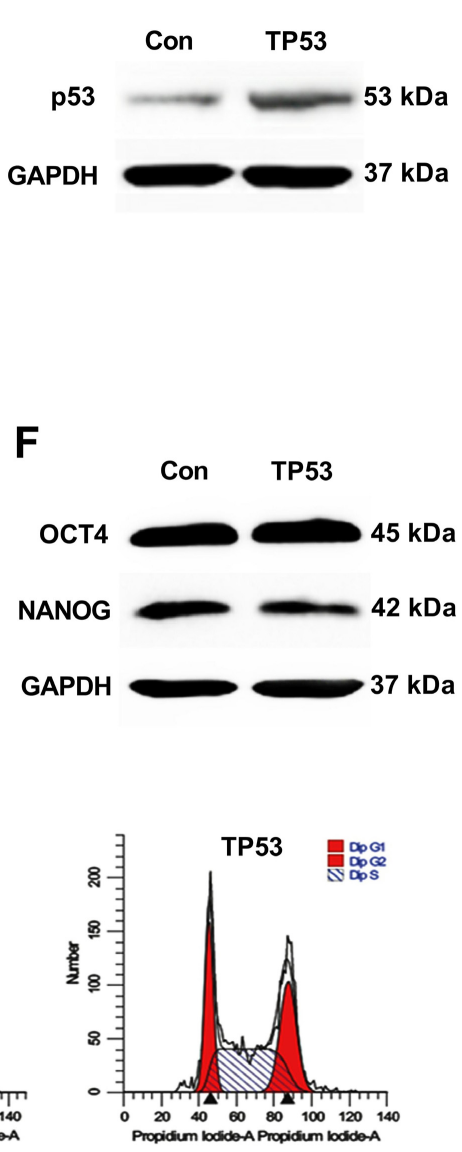

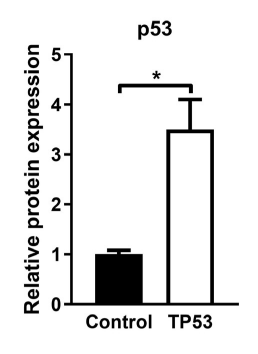

G

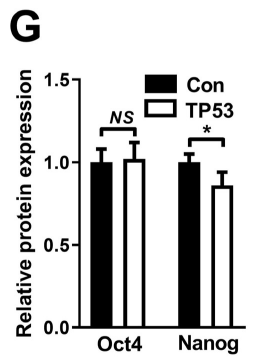

J

D

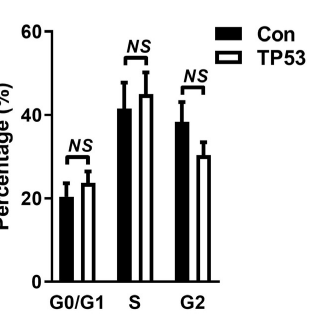

Fig. 3. Overexpression of P53 gene in hiPSC by lentivirus methods. (A) Representative images of GFP fluorescence displayed highly efficient infection of hiPSC cells in both P53 and control group. (B) The qRT-PCR results showed that hiPSC with lenti-P53 caused about 8-fold increase of P53 mRNA compared with control group. (C) Representative image of Western Blot showed significantly higher p53 protein level of lenti-P53 group than control, and quantification was done by densitometry (D). (E) The qRT-PCR results showed that overexpressed P53 had no significant effects on the mRNA expression of the pluripotency marker (OCT4, SOX2, NANOG and KLF4) of hiPSC. (F, G) The Western Blot results showed that overexpressed P53 did not change OCT4 protein level but slightly decreased Nanog with statistical significance. Representative image was shown $(\mathrm{F})$ and quantified by densitometry $(\mathrm{G})$. (H) hiPSC with lenti-P53 displayed similar mRNA levels of proliferation markers (MKI67 and AURKB). (I, J) Representative image of cell cycle analysis using flow cytometry showed cell cycle was not influenced by lenti-P53 (I) and quantification by densitometry (J). Expression values of the PCR analysis were normalized to GAPDH. Data are presented as mean \pm SD (t test, $* p<0.05$, NS not significant; $n=3$ ).

\section{p53-enhanced differentiation of cardiomyocytes depends partially on the activation of WNT3-mediated Wnt signaling pathway \\ Given that temporary activation of Wnt signaling is in-} dispensable for early mesendoderm and terminal cardiomyocytes differentiation $(11,12)$ and that WNT3 gene is a confirmed target gene of p53 transcription factor (12), loss and gain of functions of $\mathrm{p} 53$ was therefore adopted to test whether p53 could regulate the expression of WNT3. Overexpression of p53 elevated, whereas knock- down of p53 decreased, WNT3 at both mRNA (Fig. 6A and 6D) and protein (Fig. 6B, 6C, 6E and 6F) levels. Furthermore, knockdown WNT3 (Fig. $6 \mathrm{G} \sim \mathrm{I}$ ) in hiPSC significantly inhibited the p53-mediated enhancing effect of cardiomyocytes differentiation, as evidenced by decreased expression of mesendoderm markers, BRACHYURY and EOMES (Fig. 6J), cardiac mesoderm markers, MESP and KDR (Fig. 6K), cardiac progenitor marker, NKX2-5 (Fig. 6L) and cardiomyocyte marker, TNNT2 (Fig. 6M). 
A

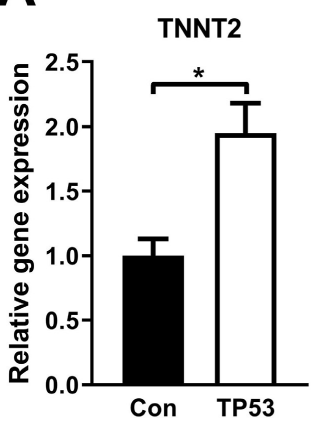

C

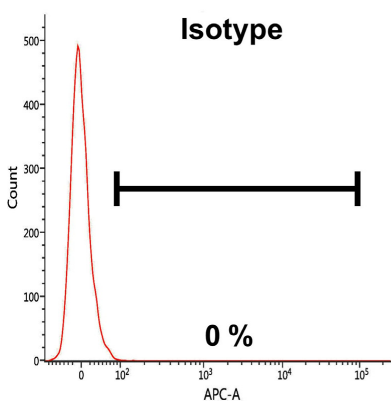

E

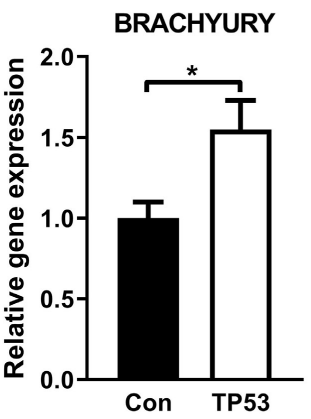

TNNI3
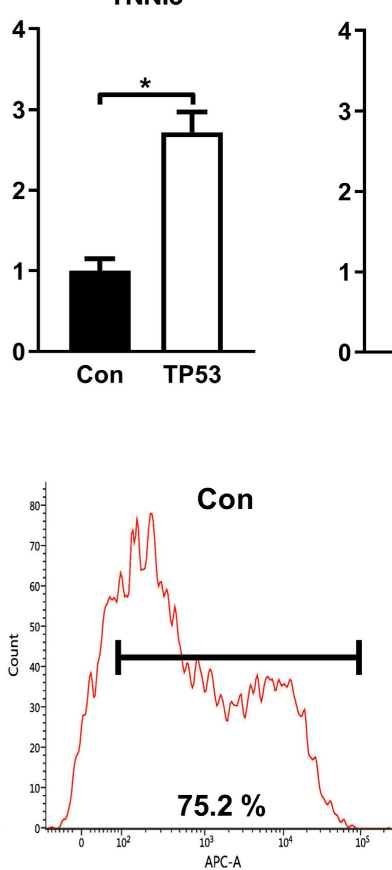

EOMES

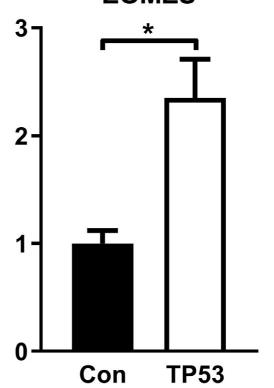

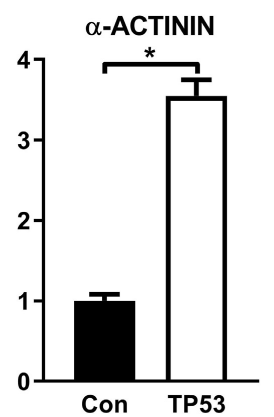

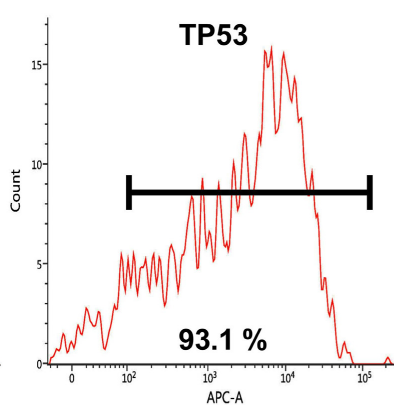

F

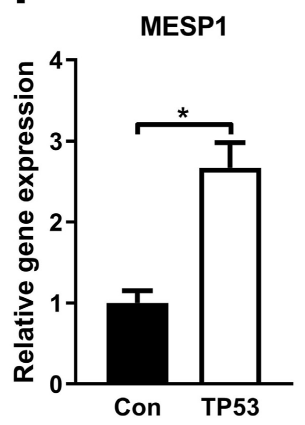

B

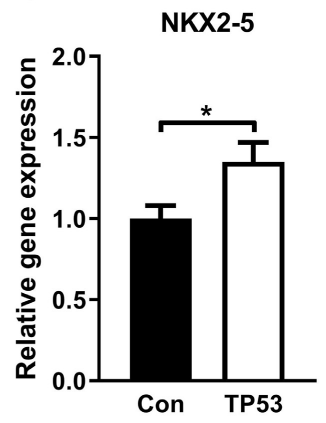

D

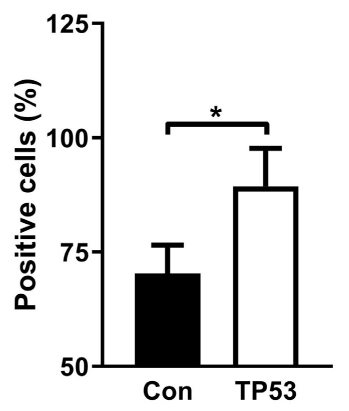

Fig. 4. p53 increases the differentiation efficiency of cardiomyocytes from hiPSC. (A, B) The qRT-PCR results showed that overexpression of P53 significantly increased mRNA levels of the cardiomyocytes markers (TNNT2, TNNI3 and $\alpha$-ACTNIN) (A) and also that of cardiac progenitor marker (NKX2-5) (B) at day 21. (C, D) Representative image of flow cytometry (C) displayed more yields of cardiomyocytes derived from hiPSC indicated by $\mathrm{CTNT}^{+}$population by lenti-P53 and quantification was performed (D). (E, F) It was showed using qRT-PCR method that p53 resulted in remarkably increased mRNA levels of mesendodermal markers (BRACHYURY and EOMES) at day 3 from differentiation (E) and cardiac mesodermal makers (MESP1 and KDR) at day $5(\mathrm{~F})$, respectively. Expression values of the PCR analysis were normalized to $\mathrm{GAPDH}$. Data are presented as mean $\pm \mathrm{SD}$ (t test, ${ }^{*} \mathrm{p}<0.05 ; \mathrm{n}=3$, performed in 3 independent experiments).

\section{p53 synergistically amplifies GSK inhibitor-mediated activation of Wnt signaling pathway through up-regulation of WNT3}

The GiWi protocol promotes differentiation of hiPSC toward cardiomyocytes through coordinated early activation by GSK inhibitor (Gi) and late inhibition by WNT inhibitor (Wi) of Wnt signaling pathway. To further define the roles of $\mathrm{p} 53$, the effect of $\mathrm{p} 53$ on the Wnt signaling was evaluated at day 1 an 5 after differentiation corresponding to the timepoint of $\mathrm{Gi}$ and Wi respectively. On day 1 post-differentiation, forced expression of P53 or WNT alone did not change the total cellular, but significantly increased the nuclear, $\beta$-catenin, an indication of the activation of the Wnt signaling pathway, and addition of GSK inhibitor further increased the p53-mediated Wnt activation (Fig. 7A and 7B). On day 5 post-differentiation, the activation of the Wnt pathway by the combined effect of p53 and WNT could be almost completely blocked by Wi. These results suggest that in the early stage (day $0 \sim 1$ ) of GiWi-mediated cardiomyocyte differ- 


\section{A}

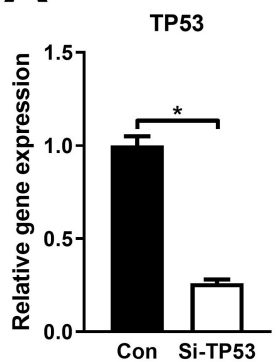

D

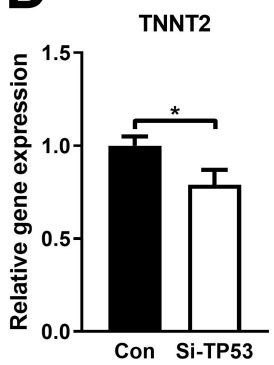

F

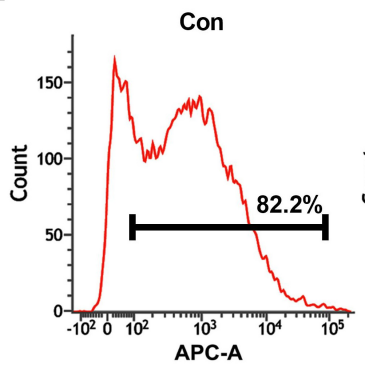

B

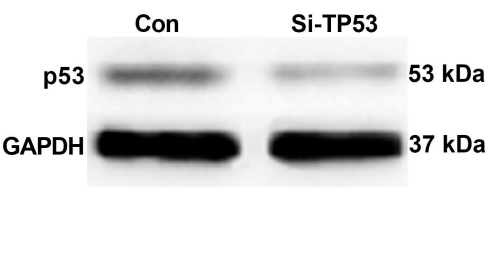

C

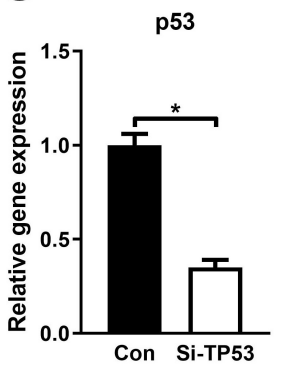

E

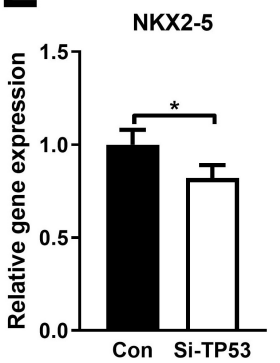

G

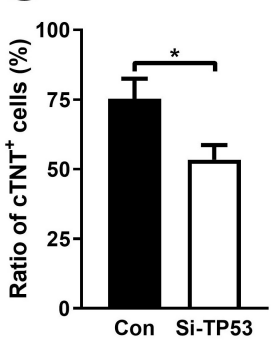

H

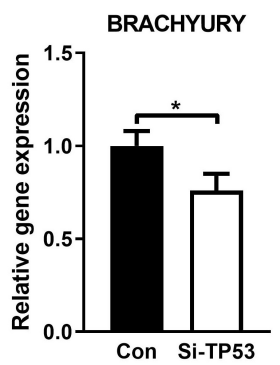

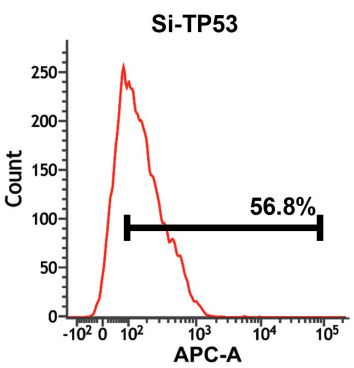
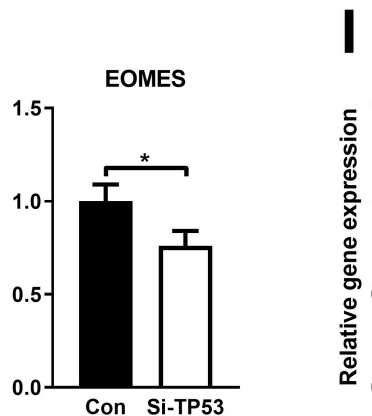
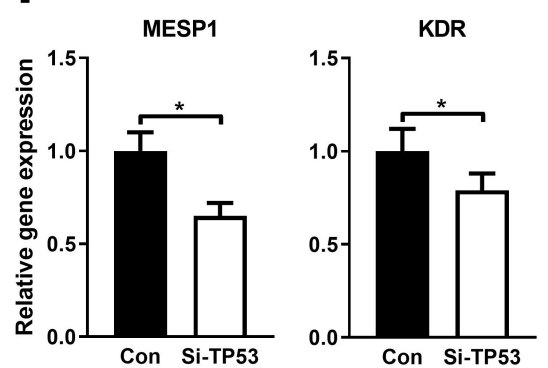

Fig. 5. Knockdown of P53 gene decreases the cardiomyocytes generation from hiPSC. (A) The qRT-PCR results showing successful knockdown of P53 in HiPSC. (B) Representative image of Western Blot displayed significantly decreased p53 protein level of lenti-Sh-P53 group (B) and quantification was done by densitometry (C). (D, E) Knockdown of P53 significantly declined mRNA levels of makers of cardiomyocytes (TNNT2) (D) and cardiac progenitor (NKX2-5) (E). (F, G) Representative image of flow cytometry (F) displayed notably decreased CTNT $^{+}$cardiomyocytes differentiated by hiPSC by knockdown of P53 in contrast with control, and quantification was done $(\mathrm{G})$. ( $\mathrm{H}$, I) The qRT-PCR results showed that knockdown of P53 markedly reduced the mRNA levels of mesendodermal markers (BRACHYURY and EOMES) at day 3 from differentiation (E) and cardiac mesodermal makers (MESP1 and KDR) at day 5 (F), respectively. Expression values of the PCR analysis were normalized to GAPDH. Data are presented as mean \pm SD ( $t$ test, ${ }^{*} p<0.05 ; n=3$, performed in 3 independent experiments). 
A

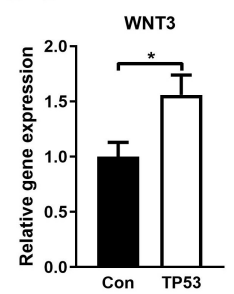

D

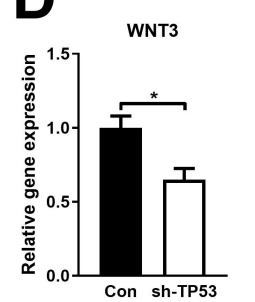

G

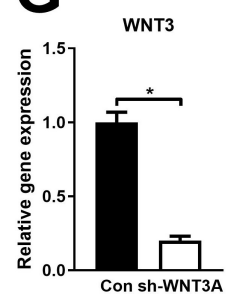

J
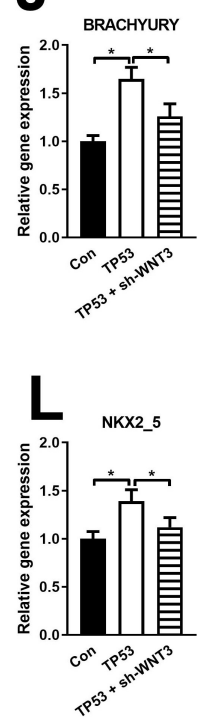

B

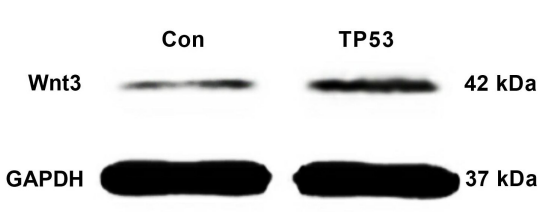

E

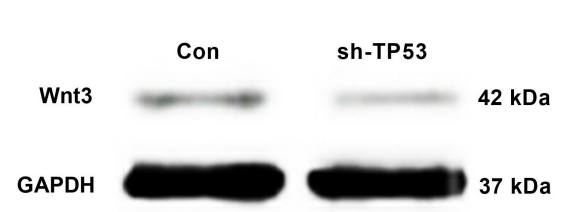

H

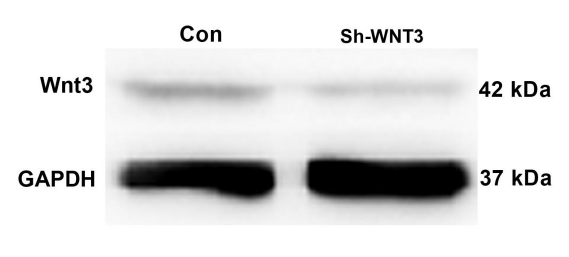

K
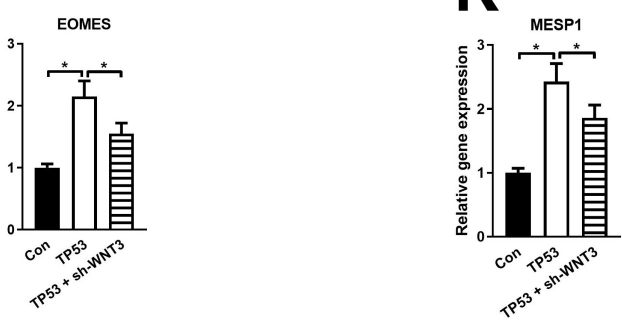

C

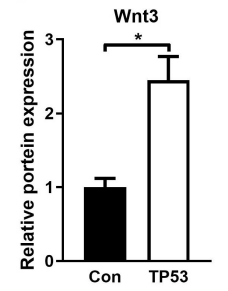

F

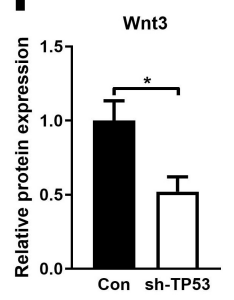

I

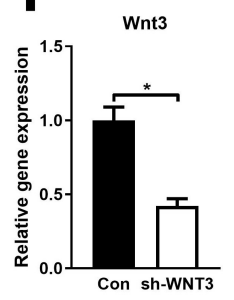

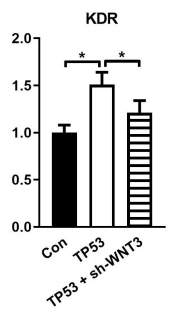

M

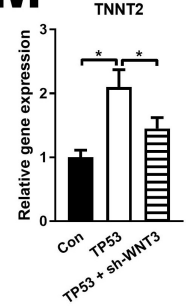

Fig. 6. Enhanced mesendodermal differentiation mediated by Wnt signaling pathway partially accounted for the effects of p53 on the cardiac differentiation from hiPSC. (A) The qRT-PCR results showing hiPSC with lenti-P53 had raised mRNA level of WNT3. It was further confirmed by representative image of Western Blot (B) and quantification by densitometry (C). (D) The mRNA level of WNT3 in hiPSC was decreased by knockdown of P53 as shown by qRT-PCR results. (E) Representative image of Western Blot displayed notably reduced protein level of WNT3 caused by knockdown of P53, and quantification was done by densitometry (G I). WNT3 gene was successfully downregulated by Sh-WNT3 using qRT-PCR (G) and Western Blot analysis (H, I). (J, K) The qRT-PCR results showed that Sh-WNT3 notably reversed P53-caused upregulation of mesendodermal markers (BRACHYURY and EOMES) (J) and cardiac mesodermal makers (MESP1 and KDR) $(K)$ at day 3 and 5 from differentiation respectively. $(L, M)$ The qRT-PCR results showed that knockdown of WNT3 significantly abrogated P53-dependent increased mRNA levels of mesendodermal markers (BRACHYURY and EOMES) at day 3 from differentiation (L) and cardiac mesodermal makers (MESP1 and KDR) at day $5(\mathrm{M})$. Expression values of the PCR analysis were normalized to GAPDH. Data are presented as mean $\pm \mathrm{SD}$ ( t test, $* \mathrm{p}<0.05 ; \mathrm{n}=3$, performed in 3 independent experiments). 
A

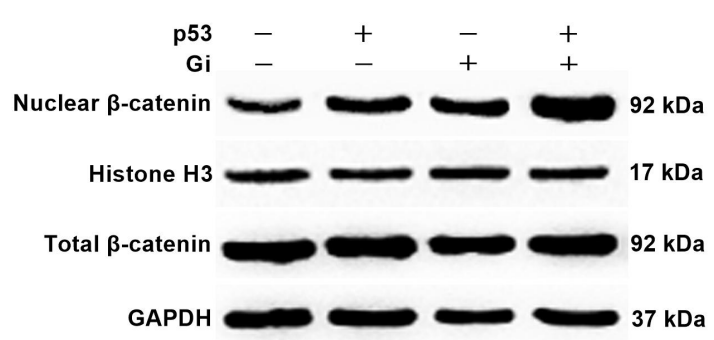

C

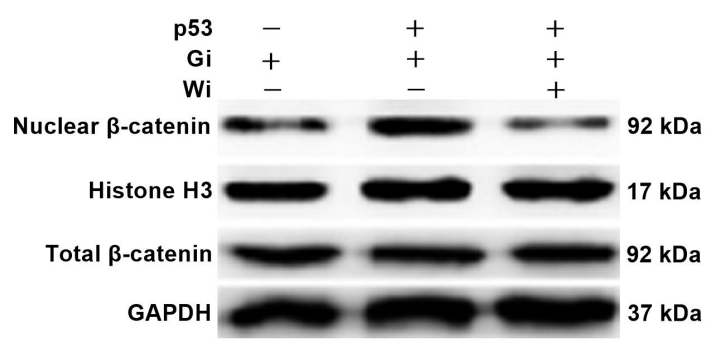

B

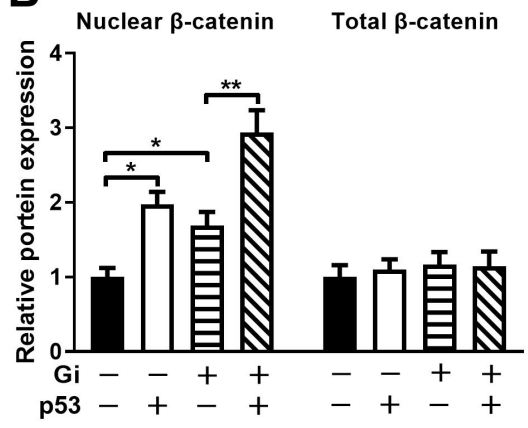

D

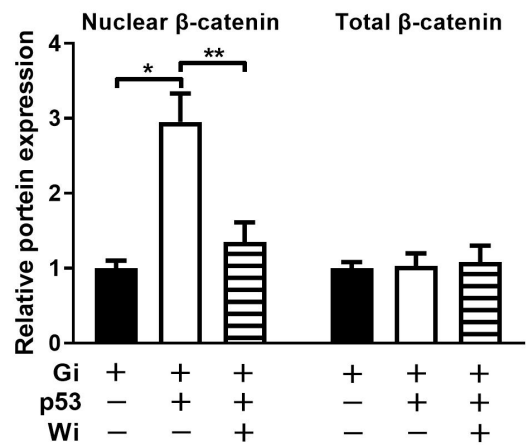

Fig. 7. Upregulated WNT3 by p53 could further amplify the activation of Wnt signaling pathway caused by GSK inhibition (Gi). However, the amplified Wnt pathway by p53 was completely blocked by Wnt inhibitor (Wi) at late stage. (A, B) The Western Blot result showing at day 1 from differentiation, overexpressing P53 or WNT3 alone could increase the localization of $\beta$-catenin in the nuclear fraction indicating activation of Wnt pathway. Moreover, P53 overexpression together with GSK inhibitor (Gi) could further amplify the activation of Gi-caused activation of Wnt signaling as shown by the representative image of Western Blot (A) and quantification by densitometry (B). (C, D) The representative image of Western Blot showed that synergically increased Wnt signaling could remarkably abrogated by WNT inhibitor (Wi) at day 5, indicated by significantly decreased nuclear $\beta$-catenin level (C) and quantification was done by densitometry (D). Expression values of the Western Blot analysis of nuclear and total protein were normalized to Histone $\mathrm{H} 3$ and GAPDH respectively. Data are presented as mean $\pm S D$ (t test, ${ }^{*} \mathrm{p}<0.05,{ }^{* *} \mathrm{p}<0.01 ; \mathrm{n}=3$, performed in 3 independent experiments).

entiation from hiPSC, the p53-induced up-regulation of WNT3 amplifies the effect of the Gi-mediated activation of Wnt signaling pathway; whereas in the subsequent Wi-mediated inhibition of Wnt signaling pathway, the effect of the p53-mediated up-regulation of WNT3 signaling is completely abolished by Wi (Fig. 7C and 7D). In other word, p53 only amplifies the effect of Gi-mediated activation of Wnt signaling at the early stage, but has no effect on the Wi-mediated inhibition of Wnt signaling at the late stage, thus realizing p53-mediated promotion of cardiomyocytes differentiation from hiPSC.

\section{Discussion}

Using the GiWi-based cardiomyocytes differentiation system in which highly efficient cardiomyocytes were induced through sequentially temporal activation and in- activation of Wnt signaling pathway (3), we found that p53 could enhance the cardiac differentiation program, resulting in remarkably higher efficiency of cardiomyocytes induction. Moreover, the promoting effect of p53 exerted at as early as mesendoderm stage, where mesendodermal specification was amplified by increased WNT3-mediated Wnt signaling.

Although well-characterized as a tumor suppressor, p53, as a transcription factor, has been shown to play crucial and as yet not fully defined functions in embryonic development and cell differentiation. The evidence that p53 participates in early development was originated from the discovery that the level of p53 expression is high in early mouse embryonic cells, and is decreased upon terminal differentiation (8). Multiple studies have then shown that p53 is implicated in cell differentiation. Normally, p53 negatively regulates proliferation and self-renewal of hem- 


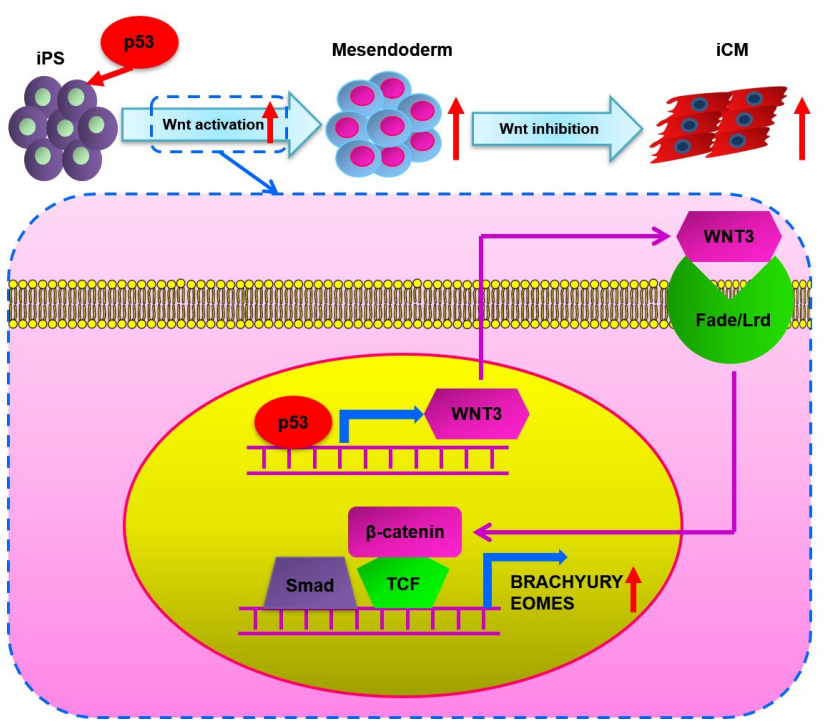

Fig. 8. Graphic abstract. Working model of how p53 enhances more cardiomyocytes generation from hiPSC. During the period from HiPSC to mesendoderm, p53 activates the Wnt signaling pathway through transactivating WNT3 expression which boosts mesendodermal differentiation, and finally yields more cardiomyocytes generation.

atopoietic and neural stem cells $(19,20)$. In the presence of specific hormones or some chemicals, p53 promotes differentiation of both mouse and human ESCs (21-23). However, in iPSC reprogramming from somatic cells, p53 inhibited the efficiency of iPSC reprogramming $(13,14)$. Further studies demonstrated that p53 plays a role in specifying mesendoderm and mesoderm. Jain et al. (21) reported that p53 preferentially promoted a mesendodermal state from hESC through activating lineageidentity genes including lncRNAs. Cordenonsi et al. (24) showed a cause-and-effect relationship between p53 and mesoderm induction in vivo, as p53-depleted xenopus embryos failed to gastrulate, whereas overexpression of p53 induces mesoderm specification. Wang et al. (12) showed that p53 family members are required for the induction of Nodal-responsive genes and for mesendoderm specification from mouse ESC. In agreement, we found that in the GiWi system of cardiomyocyte differentiation from hiPSC, p53 overexpression not only amplified the mesendoderm specification but also promoted the differentiation of its descendant cardiac progenitors and cardiomyocytes, and these effects were significantly diminished by knockdown of p53. In addition to the enhancing effect of mesendodermal specification by $\mathrm{p} 53$, we further found that p53 also impeded the ectodermal differentiation as evidenced by that Si-TP53 remarkably decreased the marker genes of ectodermal lineage (Supplementary Fig. S1B). It is pos- sible that the more potential of hiPSC toward mesendoderm by p53 results from compromised ectodermal specification, although it remains to be clarified.

Wnt signaling has been thought to be the key regulator of vertebrate heart development and particularly of cardiomyocyte differentiation (25). Lian et al. (7) established a highly efficient, stable and universal protocol for cardiomyocytes induction from hiPSC, by which sequential and temporal activation and inactivation of Wnt signaling pathway induced high yield of cardiac mesoderm and then the final induced cardiomyocytes with high efficiency. Our result showed that the enhancing effect of p53 on the cardiomyocytes generation was dependent on the Wnt signaling, as p53 increased the expression of WNT3, and more importantly, the promoting effect of $\mathrm{p} 53$ on the cardiomyocytes induction was noticeably ameliorated by simultaneous knockdown of the endogenous WNT3. Zhao et al. (26) demonstrated that optimum Wnt signaling is critical to direct the cell fate into cardiac mesoderm and increase the final efficiency and purity of derived cardiomyocytes, and lower or higher Wnt signals led to definitive endoderm or presomitic mesoderm differentiation instead of cardiac mesoderm. Therefore, upregulated and possibly fine-tuned Wnt signaling by p53 may interpret its enhancing effect of cardiomyocytes differentiation. However, the detailed mechanism remains to be elucidated in the future.

In summary, the present study has demonstrated that p53 enhances cardiomyocyte induction from hiPSC through amplification of mesendoderm specification that is mediated by WNT3-mediated Wnt signaling pathway (see graphic abstract). The findings may provide a mechanistic insight into the effect of p53 on the embryonic heart development and cardiomyocytes differentiation.

\section{Acknowledgments}

This work was supported by grants from the collaborative innovation center for prevention and treatment of cardiovascular disease of Sichuan province [xtcx-2016-18 to R. Z.].

\section{Potential Conflict of Interest}

The authors have no conflicting financial interest.

\section{Supplementary Materials}

Supplementary data including one table and one figure can be found with this article online at https://doi.org/10.15283/ijsc21051. 


\section{References}

1. Yoshida Y, Yamanaka S. Induced pluripotent stem cells 10 years later: for cardiac applications. Circ Res 2017;120: 1958-1968

2. Laflamme MA, Chen KY, Naumova AV, Muskheli V, Fugate JA, Dupras SK, Reinecke H, Xu C, Hassanipour M, Police S, O'Sullivan C, Collins L, Chen Y, Minami E, Gill EA, Ueno S, Yuan C, Gold J, Murry CE. Cardiomyocytes derived from human embryonic stem cells in pro-survival factors enhance function of infarcted rat hearts. Nat Biotechnol 2007;25:1015-1024

3. Lian X, Zhang J, Azarin SM, Zhu K, Hazeltine LB, Bao X, Hsiao C, Kamp TJ, Palecek SP. Directed cardiomyocyte differentiation from human pluripotent stem cells by modulating Wnt/ $\beta$-catenin signaling under fully defined conditions. Nat Protoc 2013;8:162-175

4. Cao N, Liang H, Huang J, Wang J, Chen Y, Chen Z, Yang HT. Highly efficient induction and long-term maintenance of multipotent cardiovascular progenitors from human pluripotent stem cells under defined conditions. Cell Res 2013; 23:1119-1132

5. Burridge PW, Matsa E, Shukla P, Lin ZC, Churko JM, Ebert AD, Lan F, Diecke S, Huber B, Mordwinkin NM, Plews JR, Abilez OJ, Cui B, Gold JD, Wu JC. Chemically defined generation of human cardiomyocytes. Nat Methods 2014;11:855-860

6. Melkoumian Z, Weber JL, Weber DM, Fadeev AG, Zhou Y, Dolley-Sonneville P, Yang J, Qiu L, Priest CA, Shogbon C, Martin AW, Nelson J, West P, Beltzer JP, Pal S, Brandenberger R. Synthetic peptide-acrylate surfaces for long-term self-renewal and cardiomyocyte differentiation of human embryonic stem cells. Nat Biotechnol 2010;28:606-610

7. Lian X, Hsiao C, Wilson G, Zhu K, Hazeltine LB, Azarin SM, Raval KK, Zhang J, Kamp TJ, Palecek SP. Robust cardiomyocyte differentiation from human pluripotent stem cells via temporal modulation of canonical Wnt signaling. Proc Natl Acad Sci U S A 2012;109:E1848-E1857

8. Schmid P, Lorenz A, Hameister H, Montenarh M. Expression of p53 during mouse embryogenesis. Development 1991;113: $857-865$

9. Lutzker SG, Levine AJ. A functionally inactive p53 protein in teratocarcinoma cells is activated by either DNA damage or cellular differentiation. Nat Med 1996;2:804-810

10. Wallingford JB, Seufert DW, Virta VC, Vize PD. p53 activity is essential for normal development in Xenopus. Curr Biol 1997;7:747-757

11. Shigeta M, Ohtsuka S, Nishikawa-Torikai S, Yamane M, Fujii S, Murakami K, Niwa H. Maintenance of pluripotency in mouse ES cells without Trp53. Sci Rep 2013;3: 2944

12. Wang Q, Zou Y, Nowotschin S, Kim SY, Li QV, Soh CL, Su J, Zhang C, Shu W, Xi Q, Huangfu D, Hadjantonakis AK, Massagué J. The p53 family coordinates Wnt and nodal inputs in mesendodermal differentiation of embryonic stem cells. Cell Stem Cell 2017;20:70-86
13. Kawamura T, Suzuki J, Wang YV, Menendez S, Morera LB, Raya A, Wahl GM, Izpisúa Belmonte JC. Linking the p53 tumour suppressor pathway to somatic cell reprogramming. Nature 2009;460:1140-1144

14. Hong $\mathrm{H}$, Takahashi $\mathrm{K}$, Ichisaka $\mathrm{T}$, Aoi $\mathrm{T}$, Kanagawa $\mathrm{O}$, Nakagawa M, Okita K, Yamanaka S. Suppression of induced pluripotent stem cell generation by the p53-p21 pathway. Nature 2009;460:1132-1135

15. Zhu J, Dou Z, Sammons MA, Levine AJ, Berger SL. Lysine methylation represses p53 activity in teratocarcinoma cancer cells. Proc Natl Acad Sci U S A 2016;113:9822-9827

16. Cordenonsi $M$, Dupont $S$, Maretto S, Insinga A, Imbriano C, Piccolo S. Links between tumor suppressors: p53 is required for TGF-beta gene responses by cooperating with Smads. Cell 2003;113:301-314

17. Lee KH, Li M, Michalowski AM, Zhang X, Liao H, Chen $\mathrm{L}, \mathrm{Xu} \mathrm{Y}$, Wu X, Huang J. A genomewide study identifies the Wnt signaling pathway as a major target of p53 in murine embryonic stem cells. Proc Natl Acad Sci U S A 2010; 107:69-74

18. Zhou R, Huang W, Fan X, Liu F, Luo L, Yuan H, Jiang Y, Xiao H, Zhou Z, Deng C, Dang X. miR-499 released during myocardial infarction causes endothelial injury by targeting $\alpha$ 7-nAchR. J Cell Mol Med 2019;23:6085-6097

19. Liu Y, Elf SE, Miyata Y, Sashida G, Liu Y, Huang G, Di Giandomenico S, Lee JM, Deblasio A, Menendez S, Antipin J, Reva B, Koff A, Nimer SD. p53 regulates hematopoietic stem cell quiescence. Cell Stem Cell 2009;4:37-48

20. Meletis K, Wirta V, Hede SM, Nistér M, Lundeberg J, Frisén J. p53 suppresses the self-renewal of adult neural stem cells. Development 2006;133:363-369

21. Jain AK, Allton K, Iacovino M, Mahen E, Milczarek RJ, Zwaka TP, Kyba M, Barton MC. p53 regulates cell cycle and microRNAs to promote differentiation of human embryonic stem cells. PLoS Biol 2012;10:e1001268

22. Li M, He Y, Dubois W, Wu X, Shi J, Huang J. Distinct regulatory mechanisms and functions for p53-activated and p53-repressed DNA damage response genes in embryonic stem cells. Mol Cell 2012;46:30-42

23. Akdemir KC, Jain AK, Allton K, Aronow B, Xu X, Cooney AJ, Li W, Barton MC. Genome-wide profiling reveals stimulus-specific functions of p53 during differentiation and DNA damage of human embryonic stem cells. Nucleic Acids Res 2014;42:205-223

24. Cordenonsi $M$, Montagner $M$, Adorno $M$, Zacchigna L, Martello G, Mamidi A, Soligo S, Dupont S, Piccolo S. Integration of TGF-beta and Ras/MAPK signaling through p53 phosphorylation. Science 2007;315:840-843

25. Foulquier S, Daskalopoulos EP, Lluri G, Hermans KCM, Deb A, Blankesteijn WM. WNT signaling in cardiac and vascular disease. Pharmacol Rev 2018;70:68-141

26. Zhao M, Tang Y, Zhou Y, Zhang J. Deciphering role of Wnt signalling in cardiac mesoderm and cardiomyocyte differentiation from human iPSCs: four-dimensional control of Wnt pathway for hiPSC-CMs differentiation. Sci Rep 2019;9:19389 